\title{
Identification of new antidiabetic agents targeting GLUT4 protein using in silico analysis
}

\author{
J. Selvaraj ${ }^{1 *}$, R. Ponnulakshmi ${ }^{2}$, V. Vishnupriya ${ }^{1}$, B. Shyamaladevi ${ }^{1}$ \\ ${ }^{1}$ Department of Biochemistry, Saveetha Dental College and Hospitals, Saveetha Institute of Medical and \\ Technical Science, Saveetha University, Chennai, Tami Nadu, India, ${ }^{2}$ Central Research Laboratory, Meenakshi \\ Academy of Higher Education and Research (Deemed to be University), Chennai, Tami Nadu, India
}

\begin{abstract}
Objective: Diabetes mellitus is a metabolic syndrome that constitutes a major health problem. It is estimated that 246 million people worldwide have diabetes and that 380 million people will be afflicted with diabetes by 2025. In addition, 3.8 million people die each year from diabetes. Natural products offer an advanced starting point in the search for highly specific and potent modulators of bimolecular function as well as novel drugs. In the present study, GLUT4 protein which plays a significant role in protecting $\beta$-cells from damage was selected as potential target. Materials and Methods: Three-dimensional structure of GLUT4 protein was build using modeller9v9.19. Modeled structure was validated through structure analysis verification server. With the purpose of identifying, the new potential drugs against GLUT4 protein molecular docking studies of 20 natural compounds were carried out using AutoDock. Results: The modeled structure has $87.9 \%$ residues in the core region. Results of docking studies clearly showed that good binding interactions of the ligand with both the targets at very low energy level. Conclusion: Based on the docking energy value, H-bond interaction the compounds hesperidin, fisetin, eriodictyol, wogonin, and chrysin was selected as the most potent compounds for GLUT4 protein. Hence, this present study suggests the consideration of these compounds for further in vitro and in vivo studies for its development as antidiabetic drugs.
\end{abstract}

Key words: Diabetics, glut4 protein, homology modeling, molecular docking

\section{INTRODUCTION}

$\mathrm{D}$ iabetes is one of serious complex conditions which can change the entire body. The main source of energy is blood glucose which is come from the food we eat. Diabetes is a disease that occurs when blood glucose is too high. The World Health Organization reports there are two types of diabetes, i.e., Type 1 diabetes (T1D) and Type 2 diabetes (T2D). T1D also called as juvenile variety of diabetes it was caused by the absolute deficiency of insulin due to the destruction of insulin-producing pancreatic $\beta$-cells. T2D is a multifactorial disease it was caused by resistance of insulin with not only hyper insulin emia and hyperglycemia but also atherosclerosis, hypertension, and an abnormal lipid profile. ${ }^{[1]}$ There are $90-95 \%$ of peoples were diagnosed with T2D. ${ }^{[2]}$ India is one among the six countries with the leading amount of diabetes patients and the every year the number was increased. According to the World Health
Organization report, India had 69.1 million diabetes cases in 2015. By 2035, the number of affected people is expected to increase to 592 million globally. About $80 \%$ of adults suffer in diabetes in low- and middle-income countries. ${ }^{[3]}$ Many reports stated that diabetes has a great impact on the Indian future economy. ${ }^{[4]}$ Hence, there is a great concern which needs to be addressed against diabetics. It also stimulates us to search the new and very effective antidiabetic agents. There are many drugs available in the market with very expensive and lots of side effects. Hence, the current situation in India and other countries increases the demand for identifying a

\section{Address for correspondence:}

Dr. Jayaraman Selvaraj, Department of Biochemistry, Saveetha Dental College, Saveetha Institute of Medical Technical Sciences, Saveetha University, Chennai, Tamil Nadu, India. Tel.: 044-26801583.

E-Mail: jselvaendo@gmail.com

Received: $13-11-2018$

Revised: $14-12-2018$

Accepted: 24-12-2018 
cost-effective antidiabetic drug with no major side effects that could be help the whole diabetic population. The World Health Organization specialist team on diabetes has listed one of its recommendations that traditional methods of treatment for diabetes should be further investigated. ${ }^{[5]}$

From thousands of year's natural products is one best alternative medicine for many diseases. ${ }^{[6]}$ In the past 200 years, there is tremendous research has been developed in the field natural product drug discovery. The plant-based drugs are still used today such as salicylic acid, digitoxin, morphine, quinine, and pilocarpine. Since ancient time, the natural products have played a significant role in the traditional treatment of T2D. ${ }^{[7]}$ The first identified diabetes dates back to the Ebers papyrus in Egypt around 1500 B.C. ${ }^{[8]}$ Later, in India, the early Ayurvedic texts such as the Sushruta Samhita and the Charaka Samhita, which were written in the $4^{\text {th }}-5^{\text {th }}$ century B.C., described the use of approximately 760 and 500 species of medicinal plants, respectively. ${ }^{[9]}$ In the present study, we aimed to discover the new plant-based drug that is used for the treatment of diabetes using computeraided drug design (CADD) approach.

CADD is one of the greatest methods significantly contributed in the field of drug discovery and development in recent years. Drug discovery and development is a timeconsuming and expensive process. On average, it takes $10-15$ y and $\$ 500-800$ million to introduce a drug into the market. ${ }^{[10]}$ In this situation CADD, approaches have been extensively used in the pharmaceutical industry to speed up the process. It helped the scientists to focus on the most promising compounds so it can be minimize the synthetic and biological testing efforts. Molecular docking is one of the computational approaches in CADD used to find out the best matches between a receptor and a ligand. It identify the conformations and orientation of the ligand within a binding site and attempts to place the ligand into the binding site in configurations and conformations appropriate for interacting with the receptor.

\section{MATERIALS AND METHODS}

\section{Datasets}

The structures of 20 compounds were downloaded from Pubchem database. The crystal structure of GLUT 4 protein was not available in protein data bank (PDB) database. Hence, the three-dimensional (3D) structure of GLUT4 was modeled using homology modeling approach.

\section{Template Search and Sequence Alignment}

In the present investigation, the protein sequence of GLUT4 was retrieved from UniProtKB/Swiss-prot database (ID P14672). ${ }^{[1]}$ The protein sequence consists of 509 amino acids. To find out the suitable for modeling, the GLUT4 protein search with default parameter was performed against the brook heaven PDB. High percentage of sequence identity and lower e-value these are two main criteria was used to select the correct template. Based on these criteria, the PDB ID 4PYP was selected as the template to generate the model, as it has the identity score of $65 \%$. Quality of the model depends on the identity between the target and template protein. Hence, the alignment between the target and template was carried out using Clustal Omega. ${ }^{[12]}$

\section{Molecular Modeling of GLUT4 Protein}

Homology modeling is a theoretical method that is used to predict the structure of protein from its sequence with an accuracy that is similar to the best results attained experimentally. Homology modeling of the GLUT4 protein was performed based on the crystal structure of 4PYP. This was used as template to build the 3D structure of GLUT 4 protein. The o template coordinate file was retrieved from the protein databank. Using the sequence alignment between the target and template, the 3D model was build using modeller9v9.19 software. ${ }^{[13,14]}$ PYMOL was used to visualize the modeled structure. ${ }^{[15]}$ All the modeled structures were ranked based on the internal scoring function (DOPE score), and those with the least internal score was selected as best model and utilized for model validation. ${ }^{[16-18]}$

\section{Assessment of the Model}

Quality of the models was assessed with respect to their energy and stereochemical geometry. ${ }^{[19]}$ The stereochemical quality of the modeled protein has been validated by the inspection of Phi/Psi distributions of Ramachandran plot from PROCHECK analysis program using structure analysis verification server (SAVS). ${ }^{[20]}$ To assess the reliability of the modeled structure of GLUT4 protein, we calculated the root mean square deviation (RMSD) by superimposing it on the template structure using a Chimera.

\section{Ligands}

The 3D structure of 20 selected compounds such as fisetin (5281614), Morin (5281670), eriodictyol (440735), hesperidin (10621), naringenin (932), apigenin (5280704), baicalein (5281605), chrysin (5281607), luteolin (5280445), tangeritin (68077), wogonin (5281703), isorhamnetin (5281654), kaempferol (5280863), rutin (5280805), quercetin (5280343), genestin (5280961), daidzen (5281708), cyanidin (441674), troxerutin (5486699), and delphinidin (68245) was retrieved as SD file from Pubchem database.

\section{Protein Preparation}

To prepare the structure of homology modeled GLUT4 protein for docking studies, the ligand and the water 
molecules were removed. Using the prepare receptor4. py script from MGLTools, charges and nonpolar hydrogen atoms were added.

\section{Ligand Preparation}

The structure of compounds was downloaded from Pubchem database. All structures were energy minimized by the MM2 method and converted to pdb extension file which is readable at the AutoDock tools (ADT) interface.

\section{Protein-ligand Interaction using AutoDock}

Using the ADT v1.5.4 and AutoDock v4.2 program, the docking analysis was carried out. ${ }^{[2]}$ To run the docking, the searching grid extended for target protein was used to and in the ligand, the polar hydrogen was added. Kollman charges were allocated and atomic solvation parameters were added. Polar hydrogen charges of the Gasteiger-type were assigned and the nonpolar hydrogen was merged with the carbons and the internal degrees of freedom and torsions were set. During the docking process, GLUT4 protein was kept as rigid and ligand allowed to move freely. Using the blind docking option, the search was extended to the whole protein. Affinity maps for all the atom types present, as well as an electrostatic map, were computed with a grid spacing of $0.375 \mathrm{~A}^{\circ}$. The Lamarckian genetic algorithm was used for docking studies. Based on the binding energy, the results were sorted. Based on the (RMSD values), a cluster analysis was carried out with reference to the starting geometry. It was subsequently performed. The lowest energy conformation of the more populated cluster was considered as the most trustable solution. ${ }^{[21]}$

\section{RESULTS AND DISCUSSION}

\section{Sequence Alignment between Template and Target}

Sequence alignment is a way of arranging the protein sequences used to recognize the area of similarity that may be a consequence of functional, structural, or evolutionary relationship between the sequences. For homology, modeling template selection and sequence alignment between the target and template are one of the main criteria. Hence, to select the template PSI - BLAST was performed. Based on the BLAST results, the PDB id: 4PYP which has $65 \%$ of similarity with target protein and has the e-value of 0.0 (0.0 indicated good similarity with template) was selected as template for further analysis. Alignment between selected target and template was carried out using Clustal Omega. The alignments which included the residues that were conserved in both the template and query sequences were shown in Figure 1. The identical residues between the query and the template sequences were exposed with the same color.

\section{Homology Modeling of GLUT4 Protein}

Homology modeling gives a clear relationship of homology between the target protein sequence and the protein sequence whose structure has been solved. The 3D structure of the protein provides a valuable insight into its molecular function helps to analyze their interactions with suitable substrates or inhibitors. As a result of homology modeling, totally five models were predicted, among them best model structure was identified based on their dope score [Table 1] which was calculated by model-single.py command in modeler program. The model TvLDH.B99990003 having minimum dope score was considered as the best model of the GLUT4 protein. The modeled structure was shown in Figure 2.

This model has similar structural features to the template protein. The $\mathrm{N}$-terminal and $\mathrm{C}$-terminal domains are recognized which provides valuable insight into molecular function and also enables the protein-protein interaction to be analyzed.

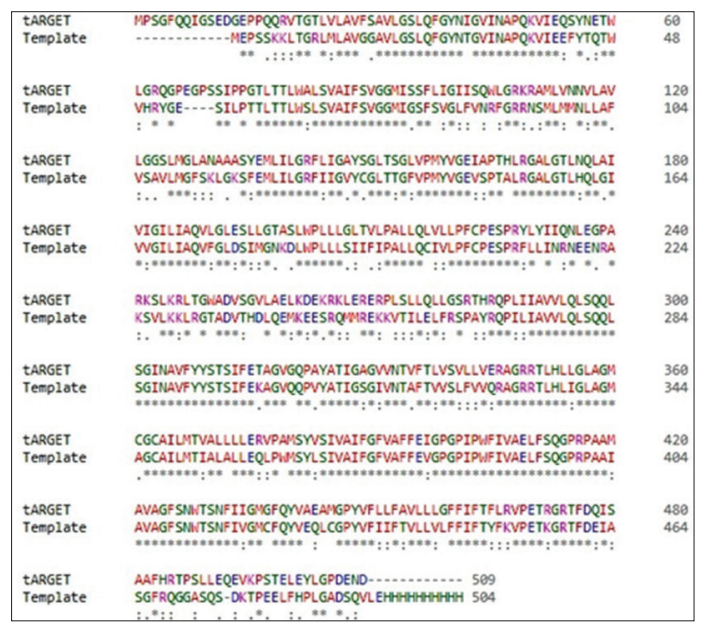

Figure 1: Alignment between target and template sequence obtained from Clustal Omega

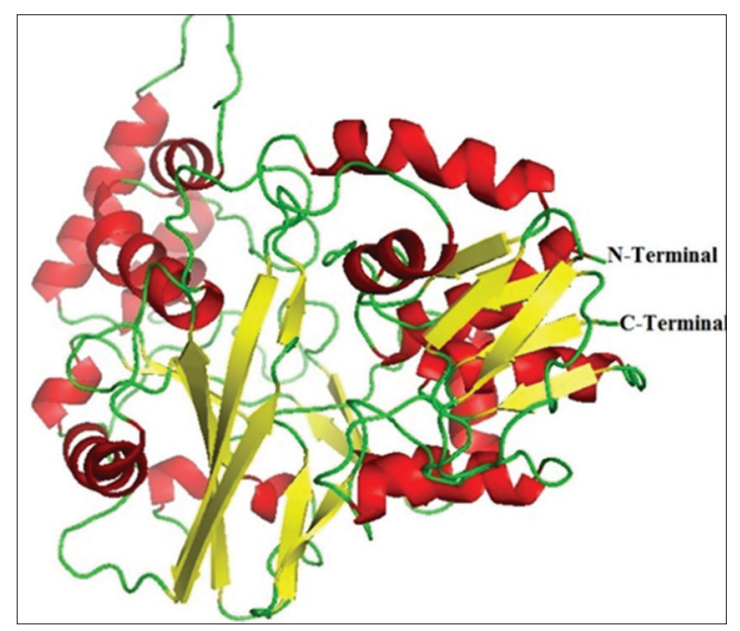

Figure 2: The best-modeled structure of GLUT 4 protein obtained from Modeler 9v9.19. Red color indicates alpha helices, yellow color indicates the beta sheets, and green color indicates the loops 


\section{Model Validation}

The modeled structures were then subjected to model validation to verify the stereochemical quality of the modeled structure. SAVS was used to validate the modeled structure. A Ramachandran plot calculation was carried out with PROCHECK program. The Phi/Psi distributions of the Ramachandran plot for TvLDH.B99990003.pdb have shown $87.9 \%$ of residues in the most favored regions, $10.3 \%$ residues in the additionally, $7 \%$ residues in generously allowed regions, and 1.0\% disallowed regions [Figure 3]. The RMSD value obtained as a result of superimposition using Chimera was $0.097 \AA$, which indicates that the generated model is quite similar to the template [Figure 4].

\section{Active Site Prediction}

Active site analysis using q site finder module reveals that the compounds were bound in the cavity of the protein containing the following residues VAL 94, TYR 110, PRO 111, VAL 112

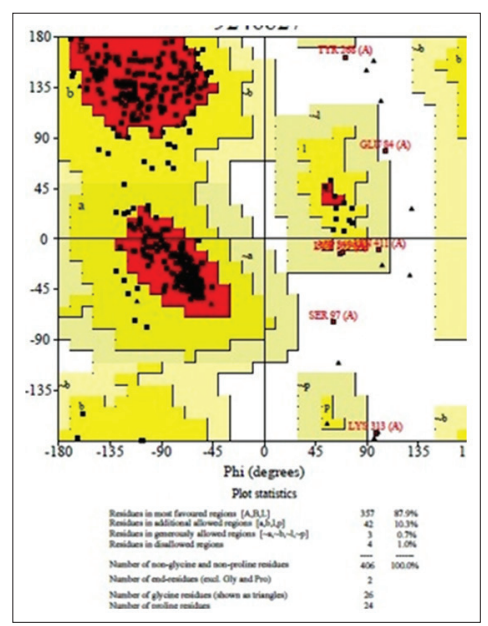

Figure 3: Ramachandran plot of GLUT4 protein obtained from PROCHECK

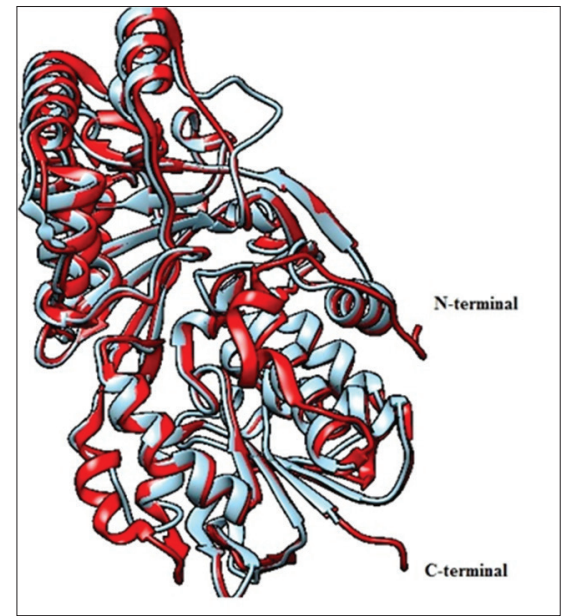

Figure 4: Superimposition of modeled GLUT4 protein (target) and template 4PYP
GLN, 113, PHE, 148, PHE 164, PHE 165, TYR 168, THR169, VAL 172, TRP 173, TRP 184, ARG 188, VAL 189, ALA 190, TYR 326, TYR 335, ARG 336, TYR 405, and TYR 439 which were involved in making hydrogen bonds with GLUT4 protein with inhibitors. These amino acids residues play a major role in the binding affinity with the ligand molecule.

\section{Molecular Docking}

To further validate the further quality of the modeled structure and understanding the binding mechanism in terms of affinity as well as selectivity, in this study, molecular docking was carried out. In the predicted active sites, the molecular docking was carried out by the AutoDock program. Results of this docking study showed that selected compounds effectively bind to active site region of GLUT4 protein and it also provides significant results by the least values of the binding energy. This also helped to identify important residues involved in GLUT4-compounds interaction. Results of docking studies showed that there are many conserved amino acid residues in GLUT4 play important role in maintaining a functional protein conformation and also directly involved in binding to GLUT4 protein. As is well known, hydrogen bonds play an essential role in the structure and function of biological molecules. In order gets the $\mathrm{H}$ bonding details, LigPlot analysis was carried out. It is used to expose the interaction mechanism between the GLUT4 and selected compounds. The best possible binding affinities and H-bond interaction details of the best five compounds at targeted protein active sites were displayed in Figure 5, and their corresponding energy values were listed in Table 2, respectively.

Analysis of results of the docking studies showed that most of the compounds strongly interact with active site of GLUT4 protein through H-bond interaction. The amino acids, namely HIS-64, VAL-112, GLN-113, TRP-173, TYR-326, ARG-336, TYR-405, and GLN-444 involved in H-bond interaction with the compound. The H-bond involvement used the complex to achieve the established conformation of the complex structure. Hydrogen bond interaction could be acted either antagonist or agonist for a ligand with receptor. In our study, hydrogen bond interaction worked as antagonist. We also noticed that most of the compounds have $>3 \mathrm{H}$-bond interaction with GLUT4 protein, it stated that more number of H-bond showed that the high affinity of ligand with receptor [Table 2]. From these docking studies, we can also able to find out the atoms involved in accepting and donating H-bond. A hydrogen bond donor defined nitrogen or oxygen atom with hydrogen attached; while an acceptor defined nitrogen, oxygen, or fluorine atom with at least one vacant valence to accept a hydrogen atom. In the present study, oxygen with hydrogen was mostly involved in donating H-bond, and nitrogen atom was generally involved in accepting H-bond. From these docking studies, we find out that HIS-64, VAL-112, GLN-113, TRP-173, TYR-326, 


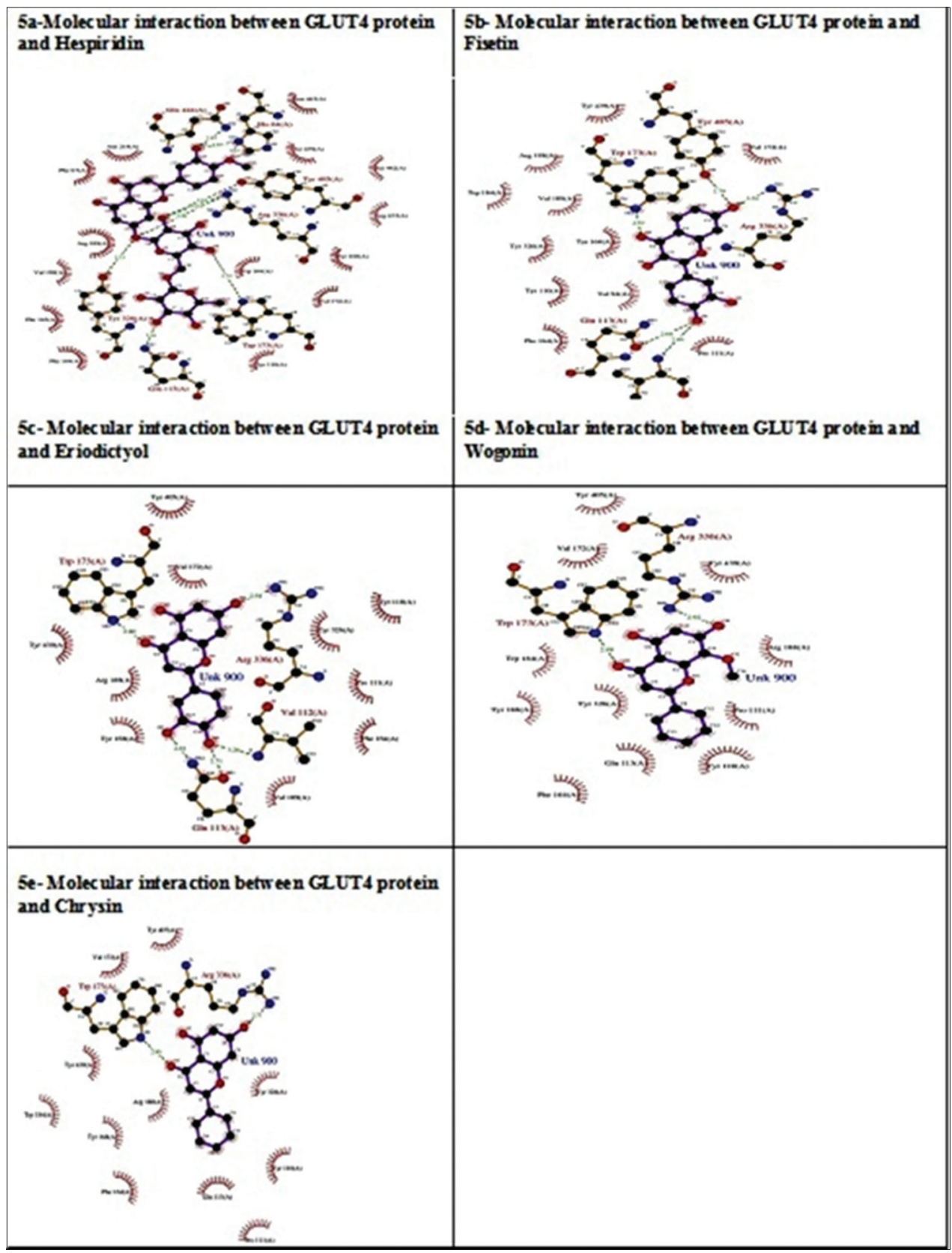

Figure 5: Molecular interactions of GLUT4 protein and top ranked ligands through $\mathrm{H}$-bond interactions

\begin{tabular}{|c|c|}
\hline File name & DOPE score \\
\hline TvLDH.B99990001.pdb & -38013.863216 \\
\hline TvLDH.B99990002.pdb & -37802.813400 \\
\hline TvLDH.B99990003.pdb & -39072.812347 \\
\hline TvLDH.B99990004.pdb & -36487.41724 \\
\hline TvLDH.B99990005.pdb & -36851.152344 \\
\hline
\end{tabular}

ARG-336, TYR-405, and GLN-444 these residues may be functionally important residues used to understand the function of protein. Of 20 compounds hesperidin, fisetin, eriodictyol, wogonin, and chrysin had the very good binding with GLUT4. Hence, these compounds may be act as lead to identify the new antidiabetic agents.

\section{CONCLUSION}

Of 20 compounds the hesperidin, fisetin, eriodictyol, wogonin, and chrysin were selected as best compounds based on the docking results. Further investigation of the function of the compound will help a better understanding the development of these compounds as an antidiabetic agent. 


\begin{tabular}{|c|c|c|c|c|c|}
\hline $\begin{array}{l}\text { Compound } \\
\text { name }\end{array}$ & $\begin{array}{l}\text { Amino acids involved } \\
\text { in interaction }\end{array}$ & $\begin{array}{l}\text { Ligand atom involved in } \\
\mathrm{H} \text {-bond interaction }\end{array}$ & $\begin{array}{l}\text { H-bond } \\
\text { distance }\end{array}$ & $\begin{array}{c}\text { Energy } \\
\text { value (kcal/mol) }\end{array}$ & $\begin{array}{c}\text { Inhibition } \\
\text { constant (nM) }\end{array}$ \\
\hline Hesperidin & $\begin{array}{l}\text { HIS - } 64 \\
\text { GLN - } 113 \\
\text { TRP - } 173 \\
\text { TYR - } 326 \\
\text { ARG - } 336 \\
\text { TYR - } 405 \\
\text { GLN - } 444\end{array}$ & $\begin{array}{l}\mathrm{ND1} \\
\mathrm{NE} 2 \\
\mathrm{NE} 1 \\
\mathrm{OH} \\
\mathrm{NH} 1 \\
\mathrm{OH} \\
\mathrm{NE} 2\end{array}$ & $\begin{array}{l}2.87 \\
3.16 \\
2.72 \\
2.73 \\
3.08 \\
2.84 \\
2.85\end{array}$ & -8.39 & 7.83 \\
\hline Fisetin & $\begin{array}{l}\text { VAL } 112 \\
\text { GLN } 113 \\
\text { TRP } 173 \\
\text { ARG } 336 \\
\text { TYR } 405\end{array}$ & $\begin{array}{l}\mathrm{N} \\
\mathrm{OE} 1 \\
\mathrm{NE} 1 \\
\mathrm{NH} 1 \\
\mathrm{OH}\end{array}$ & $\begin{array}{l}2.84 \\
2.68 \\
2.67 \\
3.24 \\
2.70\end{array}$ & -7.03 & 7.51 \\
\hline Eriodictyol & $\begin{array}{l}\text { VAL } 112 \\
\text { GLN } 113 \\
\text { TRP } 173 \\
\text { ARG } 336\end{array}$ & $\begin{array}{l}\mathrm{N} \\
\text { OE1 } \\
\mathrm{NE} 1 \\
\mathrm{NH} 1\end{array}$ & $\begin{array}{l}3.26 \\
2.71 \\
2.81 \\
2.84\end{array}$ & -6.84 & 7.38 \\
\hline Wogonin & $\begin{array}{l}\text { TRP } 173 \\
\text { ARG } 336\end{array}$ & $\begin{array}{l}\text { NE1 } \\
\text { NH1 }\end{array}$ & $\begin{array}{l}2.88 \\
2.92\end{array}$ & -5.79 & 6.65 \\
\hline Chrysin & $\begin{array}{l}\text { TRP } 173 \\
\text { ARG } 336\end{array}$ & $\begin{array}{l}\mathrm{NE} 1 \\
\mathrm{NH} 1\end{array}$ & $\begin{array}{l}2.90 \\
2.77\end{array}$ & -5.37 & 5.86 \\
\hline
\end{tabular}

\section{REFERENCES}

1. Malviya N, Jain S, Malviya S. Antidiabetic potential of medicinal plants. Acta Pol Pharm 2010;67:113-8.

2. Prabhakar PK, Doble M. A target based therapeutic approach towards diabetes mellitus using medicinal plants. Curr Diabetes Rev 2008;4:291-308.

3. ModakM,DixitP,Londhe J, GhaskadbiS, Devasagayam TP. Indian herbs and herbal drugs used for the treatment of diabetes. J Clin Biochem Nutr 2007;40:163-73.

4. Bjork S, Kapur A, King H, Nair J, Ramachandran A. Global policy: Aspects of diabetes in india. Health Policy 2003;66:61-72.

5. Howes M, Simmonds M. Plants used in the treatment of diabetes. In: Soumyanath A, editor. Traditional Medicines for Modern Times. Boca Raton: CRC Press: 2005.

6. Mosihuzzaman M, Nahar N, Ali L, Rokeya B, Khan AK, Nur-E-Alam M, et al. Hypoglycemic effects of three plants from eastern Himalayan belt. Diabetes Res 1994;26:127-38.

7. Kelley LA, Gardner SP, Sutcliffe MJ. An automated approach for clustering an ensemble of NMR-derived protein structures into conformationally related subfamilies. Protein Eng 1996;9:1063-5.

8. Shukla K, Narain JP, Puri P, Gupta A, Bijlani RL, Mahapatra SC, et al. Glycaemic response to maize, Bajra and barley. Indian J Physiol Pharmacol 1991;35:249-54.

9. Vuksan V, Sievenpiper JL. Herbal remedies in the management of diabetes: Lessons learned from the study of ginseng. Nutr Metab Cardiovasc Dis 2005;15:149-60.

10. Song CM, Lim SJ, Tong JC. Recent advances in computer-aided drug design. Brief Bioinform 2009;10:579-91.

11. Bairoch A, Apweiler R. The SWISS-PROT protein sequence database and its supplement trEMBL in 2000. Nucleic Acids Res 2000;28:45-8.

12. Altschul SF, Gish W, Miller W, Myers EW, Lipman DJ. Basic local alignment search tool. J Mol Biol 1990;215:403-10.

13. Kuntal BK, Aparoy P, Reddanna P. Easy Modeller: A graphical interface to MODELLER. BMC Res Notes 2010;3:226.

14. PyMOL. PyMOL Molecular Graphics System; 2010. Available from: http://www.pymol.org. [Last accessed on 2010 Jul 01].

15. Anupriya G, Roopa K, Basappa S, Chong YS, Annamalai L. Homology modeling and in silico screening of inhibitors for the substrate binding domain of human siah2: Implications for hypoxia-induced cancers. J Mol Model 2011;17:3325-32.

16. Sali A, Blundell TL. Comparative protein modelling by satisfaction of spatial restraints. J Mol Biol 1993;234:779-815.

17. Brooks BR, Bruccoleri RE, Olafson BD, States DJ, Swaminathan S, Karplus M. CHARMM: A program for macromolecular energy, minimization, and dynamics calculations. J Comput Chem 1983;4:187-217.

18. Hooft RW, Vriend G, Sander C, Abola EE. Errors in protein structures. Nature 1996;381:272.

19. Ramachandran GN, Ramakrishnan C, Sasisekharan V. Stereochemistry of polypeptide chain configurations. J Mol Biol 1963;7:95-9. 
20. Lengauer T, Rarey M. Computational methods for biomolecular docking. Curr Opin Struct Biol 1996;6:402-6.

21. Morris GM, Goodsell DS, Halliday RS, Huey R, Hart WE, Belew RK, et al. Automated docking using a
Lamarckian genetic algorithm and an empirical binding free energy function. J Comput Chem 1998;19:1639-62.

Source of Support: Nil. Conflict of Interest: None declared. 